\title{
Correction to: Admission to the surgical intensive care unit during intensivist coverage is associated with lower incidence of postoperative acute kidney injury and shorter ventilator time
}

\author{
Tak Kyu Oh $^{1}$ (D) In-Ae Song ${ }^{1} \cdot$ Young-Tae Jeon ${ }^{1,2}$
}

Published online: 10 October 2019

(c) Japanese Society of Anesthesiologists 2019

\section{Correction to: Journal of Anesthesia \\ https://doi.org/10.1007/s00540-019-02684-8}

Publisher's Note Springer Nature remains neutral with regard to jurisdictional claims in published maps and institutional affiliations.

In the original publication of the article, under the abstract, the last sentence of results was published incorrectly. The correct sentence should be as below.

Additionally, the median time of ventilator use in the nonintensivist coverage group was significantly longer than that in the intensivist coverage group [7.8 (interquartile range, IQR 2.6-13.8) h vs. $5.3(1.8-8.3) \mathrm{h} ; P<0.001]$.

The original article has been corrected.

The original article can be found online at https://doi.org/10.1007/ s00540-019-02684-8.

Tak Kyu Oh

airohtak@hotmail.com

1 Department of Anesthesiology and Pain Medicine, Seoul

National University Bundang Hospital, Gumi-ro 173

Beon-gil, Bundang-gu, Seongnam 13620, South Korea

2 Department of Anesthesiology and Pain Medicine, College of Medicine, Seoul National University, Seoul, South Korea 